\title{
Simposio \\ Nuevos desarRollos en el DIAGNÓSTICO DE PARÁSITOS
}

\section{Uso de PCR múltiple en el diagnóstico simultáneo de parasitosis}

\author{
José M. Rubio, Marwa A. Tammam, Thuy H. Ta-Tang \\ Laboratorio de Malaria y otras Parasitosis Emergentes, Servicio de Parasitología, \\ Centro Nacional de Microbiología, Instituto de Salud Carlos III, Madrid, España
}

Introducción

El primer problema de los servicios de salud frente a las enfermedades de tipo microbiológico es la realización de un diagnóstico sensible y eficaz en los primeros estadios de la enfermedad.

En general, los métodos clásicos de diagnóstico de las enfermedades infecciosas, especialmente en el campo de la parasitología, no están exentos de inconvenientes. Los métodos indirectos basados en la detección de anticuerpos en algunos casos no pueden diferenciar infecciones actuales de infecciones pasadas, además de ser frecuente que presenten reacciones cruzadas con otros patógenos. Los métodos directos, como cultivos - la microscopía son de efectividad relativa, debido al nivel de sensibilidad, a la complejidad de las técnicas de tinción y cultivo, a la falta de diferenciación morfológica de especies o a la subjetividad del observador. Por el contrario, los métodos directos basados en la detección del ADN del patógeno han mostrado una alta sensibilidad y especificidad, mejorando el índice de diagnósticos correctos con el beneficio para el paciente y para la sanidad pública disminuyendo el tiempo de convalecencia y las estancias hospitalarias.

\section{Amplificación genómica: PCR convencional}

La PCR (reacción en cadena de la polimerasa) se basa en la fabricación de nuevas cadenas de ADN simple a partir del original mediante enzimas específicas denominadas ADN polimerasas. Estas enzimas tienen la propiedad de realizar copias del ADN original a partir de una cadena simple de ADN siempre que tenga un iniciador (ADN de doble cadena). Los ciclos de desnaturalización y fabricación (replicación) consecutivos dan lugar a miles de copias del ADN original delimitado por los iniciadores (Saiki, et al., 1986). La PCR convencional se basa en amplificaciones mediante dos iniciadores, o cebadores, uno homólogo a la cadena 5'-3' localizado al inicio del fragmento de
ADN que se quiere amplificar y otro a la cadena 3'-5' al final del fragmento.

Actualmente, existen infinidad de métodos de diagnósticobasadosen PCRparaladeterminaciónde microorganismos en muestras humanas, en malaria el más conocido es el desarrollado por Georges Snounou (Snounou, 1996) conocido como el método de los cinco tubos. Estos métodos para patologías parasitarias como leishmaniasis, enfermedad del sueño, toxoplasmosis o criptosporidiasis han pasado del laboratorio de investigación a los hospitales y laboratorios de referencia basados generalmente en métodos de PCR anillada (nested PCR) para aumentar la sensibilidad del diagnóstico a partir de muestras clínicas (Cruz, et al., 2002). También se han incorporado o desarrollado métodos para la detección de marcadores de resistencia, virulencia y genotipificación molecular (Fuentes, et al., 2001).

Todos estos métodos tienen el inconveniente de ser individuales, es decir, nos dan una respuesta única, presencia o ausencia del patógeno buscado o del correspondiente gen que, en la mayoría de las ocasiones, no es suficiente, por lo que nuevos desarrollos de métodos basados en sistemas de detección múltiple los están sustituyendo.

\section{Amplificación genómica: PCR múltiple}

Las PCR múltiples son aquellas, en general, que en el proceso de amplificación participan más de dos iniciadores amplificando en un único tubo varias secuencias dianas, permitiendo la detección e identificación simultánea de distintos genes (Méndez-Álvarez y Pérez-Roth, 2004). En los últimos años estos métodos han proliferado en la literatura científica pero sus inicios son paralelos a la utilización de la PCR como herramienta para el diagnóstico a mediados de los años 90 del siglo pasado.

Podrían clasificarse en dos tipos:

i) aquellas que son PCR simples reunidas en un único proceso y en un único tubo y donde 
no existe competencia entre los iniciadores. En este grupo se incluirían PCR con control interno de reacción basado en otra pareja de iniciadores (Fuentes, et al., 1996). Estos se pueden hacer tan complejos como se desee, incluyendo numerosas parejas de iniciadores específicos para sus respectivas dianas, cuya mayor delimitación es la posibilidad de diferenciar los fragmentos obtenidos, generalmente por tamaño en geles de agarosa (Tenorio, et al., 1996), y

ii) aquellas que los iniciadores compiten entre ellos.

Semi-nested múltiple malaria PCR: un ejemplo

La malaria es producida por protozoos parásitos del género Plasmodium. Cuatro especies afectan tradicionalmente a los humanos, $P$. falciparum, la forma más grave, $P$. vivax, $P$. ovale y $P$. malariae. Aunque son posibles las infecciones por otras especies ( $P$. knowlesi)

En malaria es muy importante alcanzar un diagnóstico de especie correcto ya que la seriedad, patogenia y tratamiento de la enfermedad está estrechamente relacionada con la especie - especies de Plasmodium involucradas. El diagnóstico primario se hace por microscopía $y$, más recientemente, con el apoyo de pruebas de diagnóstico rápido. Ambos métodos tienen sus dificultades, además, adolecen de sensibilidad y la caracterización de infecciones mixtas es complicada. El desarrollo de métodos moleculares intentaba suplir estas carencias. Se diseñaron diversos métodos, casi todos ellos basados en el gen ssurDNAI por dos razones; la sucesión de zonas muy conservadas y otras altamente variables, y la segunda que se disponía de un amplio número de secuencias en las bases de datos para diferentes especies de Plasmodium.

Dos estrategias se siguieron en el diseño, PCR individuales para cada Plasmodium humano
(Snounou, 1996) o sistemas de detección múltiple (Rubio, et al., 1999).

Este sistema consiste en dos amplificaciones múltiple encadenadas. La primera amplificación permite caracterizar Plasmodium sp. y, además, tiene un control interno de reacción. La segunda amplificación es una múltiple que permite identificar por tamaño del producto amplificado las cuatro especies de Plasmodium humanas (figura 1).

En la primera PCR, la pareja PLF-UNR amplifica el fragmento del gen en caso de que haya presencia de ADN de cualquier especie de Plasmodium, mientras que la pareja HUF-UNR amplifica un fragmento de 230 pares de bases correspondiente al fragmento del gen de humanos que se utiliza para comprobar que la reacción no se ha inhibido (control interno). En la segunda PCR, dependiendo de las especies involucradas en la infección, el producto de amplificación será de diferente tamaño: $250 \mathrm{pb}$ para $P$. malariae, 399 pb para $P$. falciparum, $450 \mathrm{pb}$ para $P$. ovale y 500 pb para $P$. vivax.

Las ventajas de este método de diagnóstico son su sensibilidad y su especificidad (Ataei, et al., 2011) pero, especialmente, la caracterización de las cuatro especies de Plasmodium que afectan a humanos en solo dos procesos de amplificación múltiple.

\section{Diseño de una PCR múltiple: complejidad y dificultades}

Diseñar PCR múltiple parece una tarea sencilla, en principio, la mayor dificultad parece únicamente el aumento del número de iniciadores que se deben diseñar. Pero la realidad es mucho más compleja. Entre otros factores es importante considerar los siguientes:

1. Se debe escoger un gen o regiones de genes con zonas variables y otras altamente conservadas del que se disponga de información en muchas o todas las especies de interés para nuestro objetivo.

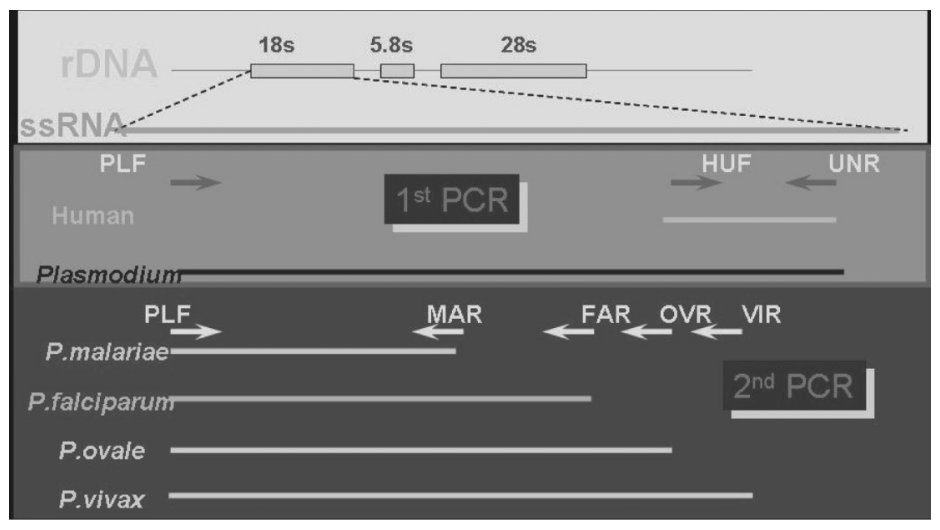




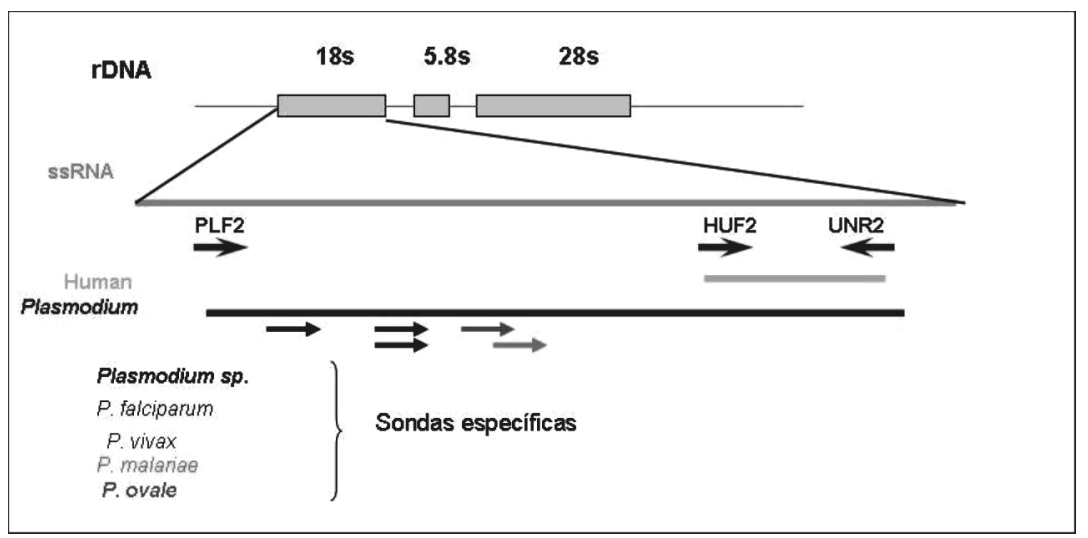

2. Se deben localizar suficientes regiones del gen diana específicas para cada especie pero que, a su vez, el tamaño del fragmento teóricamente amplificado con las respectivas parejas de iniciadores se puedan distinguir con facilidad en geles de agarosa o el método de visualización escogido.

3. El diseño de los iniciadores debe cumplir las reglas mucho más estrictamente que para el diseño de PCR convencionales (Rubio, et al., 2002)

4. La temperatura de los iniciadores debe ser exactamente la misma para todos, en casos donde sea complejo la temperatura de alineamiento debe de ser compensada por la concentración de cada uno de ellos en la mezcla de amplificación.

Además, hay que considerar que pequeñas modificaciones de la concentración de sales en la reacción, incluyendo la concentración de Mg, pueden variar y descompensar los resultados, esto afecta directamente al uso cambio de enzimas y casas comerciales. La transferencia de este tipo de métodos entre laboratorios y su optimización es mucho más compleja que la trasferencia de métodos de PCR simples.

\section{Otros sistemas múltiples en el diagnóstico parasitológico}

Los sistemas múltiples no se circunscriben únicamente a sistemas de PCR convencional con múltiples iniciadores. El avance de los métodos moleculares ha permitido desarrollar métodos basados en nuevas tecnologías como son:

1. métodos de PCR a tiempo real, bien con sistemas de iniciadores múltiples o métodos mediante la detección de múltiples sondas (figura 2) (Ta, et al., 2010),

2. métodos de microarray o microchip mediante la fijación de múltiples sondas en soportes fijos (Palacios, et al., 2007), o
3. métodos de pirosecuenciación de fragmentos previa PCR universal y fijación por múltiples sondas de los fragmentos amplificados (Sreekumar, et al., 2005).

\section{Ventaja de los sistemas múltiples}

La principal ventaja de estos sistemas es poder agrupar en un único proceso sistemas que antes requerían un número mayor de pasos, simplificando el proceso, ahorrando costes en tiempo, en personal y en recursos y lo que es más importante en diagnóstico, acortar el tiempo de respuesta.

\section{Referencias}

1. Ataei $\mathrm{S}$, Nateghpour M, Hajjaran H, Edrissian GH, Foroushani AR. High specificity of semi-nested multiplex PCR using dried blood spots on DNA Banking Card in comparison with frozen liquid blood for detection of Plasmodium falciparum and Plasmodium vivax. J Clin Lab Anal. 2011;25:185-90.

2. Cruz I, Cañavate $C$, Rubio JM, Morales MA, Chicharro $\mathrm{C}$, Laguna $\mathrm{F}$, et al. A nested polymerase chain reaction (Ln-PCR) for diagnosing and monitoring Leishmania infantum infection in patients co-infected with human immunodeficiency virus. Trans R Soc Trop Med Hyg. 2002;96(Suppl.1):S185-9.

3. Fuentes I, Rubio JM, Ramírez C, Alvar J. Genotypic characterization of Toxoplasma gondii strains associated with human toxoplasmosis in Spain: direct analysis from clinical samples. J Clin Microbiol. 2001;39:1566-70.

4. Fuentes I, Rodríguez M, Domingo CJ, del Castillo F, Juncosa T, Alvar J. Urine sample used for congenital toxoplasmosis diagnosis by PCR. J Clin Microbiol. 1996;34:2368-71.

5. Méndez-Alvarez S, Pérez-Roth E. Multiplex PCR in clinical microbiology. Enferm Infecc Microbiol Clin. 2004;22:183-91.

6. Palacios G, Quan PL, Jabado OJ, Conlan S, Hirschberg $\mathrm{DL}$, Liu $\mathrm{Y}$ et al. Panmicrobial oligonucleotide array for diagnosis of infectious diseases. Emerg Infect Dis. 2007;13:73-81.

7. Rubio JM, Benito A, Roche J, Berzosa PJ, García ML, Micó M, Edú M, Alvar J. Semi-nested, multiplex 
polymerase chain reaction for detection of human malaria parasites and evidence of Plasmodium vivax infection in Equatorial Guinea. Am J Trop Med Hyg. 1999;60:183-7.

8. Rubio JM, Post RJ, van Leeuwen WM, Henry MC, Lindergard G, Hommel M. Alternative polymerase chain reaction method to identify Plasmodium species in human blood samples: the semi-nested multiplex malaria PCR (SnM-PCR). Trans R Soc Trop Med Hyg. 2002;96(Suppl.1):S199-204.

9. Saiki RK, Bugawan TL, Horn GT, Mullis KB, Erlich HA. Analysis of enzymatically amplified betaglobin and HLA-DQ alpha DNA with allele-specific oligonucleotide probes. Nature. 1986;324:163-6.
10. Snounou G. Detection and identification of the four malaria parasite species infecting humans by PCR amplification. Methods Mol Biol. 1996;50:263-91.

11. Sreekumar C, Hill DE, Miska KB, Vianna MC, Yan L, Myers RL, Dubey JP. Genotyping and detection of multiple infections of Toxoplasma gondii using Pyrosequencing. Int J Parasitol. 2005;35:991-9.

12. Ta TT, Salas A, Ali-Tammam M, Martínez Mdel C, Lanza M, Arroyo E, Rubio JM. First case of detection of Plasmodium knowlesi in Spain by real time PCR in a traveller from Southeast Asia. Malar J. 2010;9:219.

13. Tenorio A, Echevarría JE, Casas I, Echevarría JM, Tabarés E. Detection and typing of human herpesviruses by multiplex polymerase chain reaction. J Virol Meth. 1993;44:261-9.

\title{
Uso de herramientas moleculares en el diagnóstico de parasitosis: PCR en tiempo real, iniciativas en enfermedad de Chagas
}

\author{
M. M. C. Bisio' ${ }^{1}$, C. Cura' ${ }^{1}$, T. Duffy', M. Sued ${ }^{2}$, Y. Qvarnstrom³ ${ }^{3}$ A. Da Silva ${ }^{3}$, L. Orellana², \\ A. G. Schijman ${ }^{1}$ \\ ${ }^{1}$ Laboratorio de Biología Molecular de la Enfermedad de Chagas, Instituto de Ingeniería Genética y \\ Biología Molecular-CONICET, Buenos Aires, Argentina \\ 2 Instituto de Cálculo, Universidad de Buenos Aires, Buenos Aires, Argentina \\ ${ }^{3}$ Division of Parasitic Diseases and Malaria, Center for Global Health, Centers for Disease Control \\ and Prevention, Atlanta, GA, USA
}

\section{Evaluación internacional de métodos de PCR para diagnóstico de Trypanosoma cruzi}

En un consorcio formado por INGEBI-CONICET, (CeNDIE) ANLIS Dr. Carlos G. Malbrán; CIMPAT, Universidad de los Andes (Bogotá, Colombia), Laboratorio de Biologia Molecular e Doenças Endêmicas, Instituto Oswaldo Cruz/ FIOCRUZ (Rio de Janeiro, Brasil) y Laboratorio de Serodiagnóstico para Enfermedad de Chagas, Universidade Federal de Goias (Goiania, Brasil), con el apoyo de WHOTDR y PAHO, organizamos un estudio multicéntrico para evaluar protocolos de PCR para diagnóstico molecular de infección por Trypanosoma cruzi.

En este estudio intervinieron 29 centros y laboratorios de Argentina, Bolivia, Brasil, Chile, Colombia, Guyana Francesa, México, Perú, Venezuela, Uruguay, Estados Unidos, España, Bélgica, Francia y Reino Unido. A cada uno de ellos se les enviaron tres paneles de muestras a ciegas $(n=70)$ para que sean procesadas siguiendo sus métodos de rutina: un panel formado por diluciones seriadas de ADN de cepas de referencia de $T$. cruzi, X-10 (Tc I), Cl Brener (Tc VI) y CAN III (Tc IV) y controles negativos, otro panel formado por sangre no infectada contaminada con diluciones de parásitos de cultivo y un tercer panel formado por muestras seropositivas y seronegativas de individuos de Argentina, Bolivia y Brasil.
Se informaron 48 métodos de PCR por 27 laboratorios participantes, usando como blancos moleculares secuencias de minicírculo, ADN satélite, ARN ribosómico, secuencias del miniexón y mitocondriales, usando termociclados convencionales o PCR en tiempo real. De este estudio multicéntrico se seleccionaron cuatro métodos de PCR que arrojaron los mejores resultados de especificidad y sensibilidad en los tres paneles, entre ellos dos métodos de PCR en tiempo real dirigidos a secuencias de ADN satélite, uno revelado por Sybr Green y el otro empleando la sonda TaqMan (Schijman, et al., 2011).

En primer lugar, se evaluó la reproducibilidad de estos métodos en el Laboratorio de Biología Molecular de la Enfermedad de Chagas en INGEBI, mediante un experimento en el cual cuatro operadores distintos evaluaron a ciegas, las mismas ocho muestras (muestras positivas con diferente nivel de parasitemia y muestras no infectadas), confirmándose el desempeño obtenido en el estudio multicéntrico (Schijman, et al., 2011).

En el marco de un taller realizado en INGEBI con financiación de TDR y UNU-BIOLAC en noviembre de 2008, 18 operadores representantes de los laboratorios participantes en el ensayo multicéntrico, manipularon un panel de muestras con distinta carga parasitaria y evaluaron la 
sensibilidad y la especificidad de la PCR en tiempo real revelada con SybrGreen. La especificidad y sensibilidad de esta PCR en tiempo real fue de $88 \%$ y $100 \%$, respectivamente cuando la extracción del ADN había sido por solventes orgánicos y de 100\% y $78 \%$, respectivamente cuando la extracción fue por columna de sílica gel. El taller permitió definir mejores prácticas de PCR y armonizar los procedimientos y controles de calidad entre todos los laboratorios interesados.

\section{Determinación de la carga parasitaria por PCR en tiempo real}

Hemos desarrollado un sistema cuantitativo de PCR en tiempo real para determinar la carga parasitaria en muestras de sangre periférica usando como blanco molecular la secuencia satélite e iniciadores modificados para aumentar la capacidad de reconocimiento de los distintos linajes parasitarios. Para mejorar la precisión de la cuantificación hemos incorporado al ensayo un plásmido recombinante lineal como estándar interno (IAC) que se agrega a la muestras de sangre original y se utiliza para normalizar el valor crudo de la carga parasitaria, según el rendimiento de la extracción de ADN de la muestra sanguínea y la eficiencia de amplificación (Duffy, et al., 2009).

Este procedimiento fue ensayado en el seguimiento de 38 pacientes pediátricos en tratamiento con benznidazol. También fue usado para seguir la dinámica de la parasitemia en pacientes sometidos a trasplante cardiaco con riesgo de reactivación por inmunosupresión. Esta metodología fue evaluada en estudios con muestras de tejido en modelo experimental (Zago, et al., 2008) y actualmente en pacientes con trasplante renal.

Actualmente, se realizan ensayos para evaluar la precisión de la $\mathrm{PCRq}$, según las recomendaciones del Clinical and Laboratory Estándar Institute de Estados Unidos (CLSI, 2004) utilizando sondas TaqMan en versión múltiple, que incluye la amplificación simultánea del IAC, para poder ser utilizada en los procesos de validación como marcador de eficacia en la respuesta parasitológica durante los estudios de nuevos fármacos candidatos (Convenio CONICET-Drug and Neglected Diseases Initiative, Médicos sin Fronteras).

Asimismo, en julio de 2011 se lanzará una iniciativa para la armonización y validación multicéntrica de técnicas de PCR en tiempo real para determinar la carga parasitaria en pacientes con Chagas crónico, lanzada por la Organización Panamericana de la Salud.
Detección de unidades discretas de tipificación de Trypanosoma cruzi por PCR múltiple en tiempo real con sondas TaqMan

Se diseñó un algoritmo de PCR múltiple para discriminar entre las seis unidades de tipificación discreta de $T$. cruzi (UDT), denominadas Tcl a TcVI en el marco de un consenso alcanzado en el segundo simposio satélite de nomenclatura de $T$. cruzien Buzios, Brasil, en agosto de 2009 (Zingales, et al., 2009). Se diseñaron iniciadores y sondas TaqMan hacia la región intergénica del miniexón hacia genes para ARN ribosómico $18 \mathrm{~S}$ y secuencia codificantes de la citocromo oxidasa II mitocondrial, en base al alineamiento de secuencias disponibles en GeneBank. Hasta el momento se estandarizó la PCR múltiple para secuencias intergénicas de miniexón, que permite distinguir Tc1, Tc II/V/VI, Tc III y Tc IV (Duffy, 2010). Esta estrategia fue evaluada con 27 stocks de referencia, provenientes de Argentina, Chile, Brasil, Colombia, México y Estados Unidos, pertenecientes a las seis UDT.

Con esta metodología se han caracterizado 31 aislamientos naturales de $T$. cruzi de triatominos silvestres (Triatoma gerstaeckeri, $T$. protacta, $T$. indictiva, $T$. sanguisuga y $T$. lecticularia), aislamientos de reservorios silvestres del sur de Estados Unidos, 4 de ellos compuestos por infecciones mixtas de Tc I y Tc IV y muestras clínicas de pacientes con reactivación de la enfermedad de Chagas por inmunosupresión posterior a trasplante de órganos, y se encontró Tc I y Tc V en chagomas epidérmicos de pacientes argentinos.

Financiación: PICT 33955, Agencia Nacional de Ciencia y Tecnología, PIP 112-2008-01-02915, CONICET.

\section{Referencias}

1. CLSI/NCCLS. Evaluation of precision performance of quantitative methods; Approved guideline. Second edition. CLSI document EP5-A2; 2004.

2. Duffy $\mathrm{T}$, Bisio, M, Burgos JM, Díez M, Levin MJ, Favaloro RR, et al. Accurate real-time PCR strategy for monitoring bloodstream parasitic loads in Chagas disease patients. PLoS Negl Trop Dis. 2009;3:1-10.

3. Duffy T. Búsqueda y caracterización de marcadores moleculares para identificación de linajes de $T$. cruzi en vectores, reservorios animales y pacientes infectados (tesis). Buenos Aires: Universidad de Buenos Aires; 2010.

4. Schijman $A G$, Bisio $M$, Orellana $L$, Sued $M$, Duffy T, Mejía-Jaramillo AM, et al. International study to evaluate PCR methods for detection of Trypanosoma cruzi DNA in blood samples from Chagas disease patients. PLoS Negl Trop Dis. 2011;5:e931. 
5. Zago MP, Barrio AB, Cardozo RM, Duffy T, Schijman $A G$, Basombrío MA. Impairment of infectivity and immunoprotective effect of a LYT1 null mutant of Trypanosoma cruzi. Infect Immun. 2008;76:443-51.
6. Zingales B, Andrade SG, Briones MR, Campbell DA, Chiari E, Fernández $O$, et al. A new consensus for Trypanosoma cruzi intraspecific nomenclature: second revision meeting recommends $\mathrm{Tcl}$ to $\mathrm{TcVI}$. Mem Inst Oswaldo Cruz. 2009;104:1051-4.

\title{
Nanobodies, the next generation antibody products for research, diagnosis and therapy
}

\author{
Serge Muyldermans \\ Laboratory of Cellular and Molecular Immunology, Vrije Universiteit Brussel, Brussel, Belgium
}

\section{Nanobodies and how to obtain them}

Llamas and camels have unique antibodies circulating in their blood. In contrast to classic antibodies as found in all jawed vertebrates, including human, mice and rabbits these antibodies lack a light chain and consist of a homodimer of a heavy chain polypeptide. The antigen-binding fragments of these heavy-chain antibodies are easily cloned and expressed recombinantly in microbial systems. Antigen-specific recombinant 'single domain' antibody fragments, also referred to as nanobodies because of their nanometer size of $4 \mathrm{~nm}$ by $2.5 \mathrm{~nm}$ in diameter, are obtained after a standard immunization of a dromedary or llama followed by cloning the nanobody repertoire. The in vivo affinity matured, antigen-binding nanobodies from this cloned nanobody repertoire are readily selected by phage display.

\section{Properties of nanobodies}

The recombinant, monoclonal nanobody is well produced in bacteria, is very stable and highly soluble, and it binds its cognate antigen with high affinity and specificity. Since the antigen is recognized by a single domain protein with molecular weight (MW) of only 15,000 , very often the nanobody recognizes an epitope that is difficult to target with human or mouse antibodies of 150,000 MW where the antigen-binding fragment comprises at least two domains with a total MW of 30,000 . The 'humanization' of a camel derived single domain antibody is straightforward. Probably, the largest advantage of nanobodies comes from their strict monomeric behavior and the ease to tailor them into larger pluripotent constructs (Muyldermans, et al., 2009).

\section{Nanobodies as a research tool}

In the past years we have been exploring the application range of the nanobodies. After an immobilization on a solid support, we employ the 'nano-trap' as a research tool to immune-capture the antigen from complex mixtures (Rothbauer, et al.,
2008). The intracellular expression of a nanobody fused with red fluorescent protein or 'chromobody' appears to be a potent probe to trace and visualize the antigen within living cells (Rothbauer, et al., 2006).

\section{Diagnostic nanobodies for non invasive imaging}

We demonstrated that the nanobodies, after being labeled with ${ }^{99 \mathrm{~m}} \mathrm{Tc}$, are excellent tools for in vivo imaging in mice models. Therefore, we now identified a nanobody against HER-2, a tumor associated surface marker of some breast cancer cells (Vaneycken, et al., 2011). We are currently producing this nanobody under good manufactuing practice conditions and hope that we will be able to use this nanobody to screen breast cancer patients to identify those that will benefit from treatment with traztuzmab.

\section{Nanobodies in therapy}

In another set-up, the nanobodies against a tumor specific antigen or an African trypanosome parasite were coupled with an enzyme or a truncated human ApoL1, respectively (Cortez-Retamozo, et al., 2004; Baral, et al., 2006). The former construct allows the eradication of tumors in the presence of a prodrug, according to an antibody dependent enzyme prodrug therapy (ADEPT), whereas the trypanolytic effect of the latter managed to eliminate effectively a trypanosome infection in mice models.

Finally, for our serotherapeutic nanobody project we selected nanobodies against each one of the two most toxic compounds of scorpion venom, the Aahl and Aahll toxin (Hmila, et al., 2010). The most potent neutralizing nanobody for the Aahl and that for Aahll were fused in a single polypeptide construct. This bispecific nanobody NbF12-10 was well expressed in bacterial cultures and it was demonstrated that its intravenous injection, 15 minutes after a subcutaneously administered toxic dose of the scorpion venom could protect the mouse. The potent neutralization of the crude venom 
(containing both toxins) by the bispecific nanobody exceeds the Fab'2 based horse antivenom that is currently used to treat envenomed patients.

\section{References}

1. Baral TN, Magez S, Stijlemans B, Conrath K, Vanhollebeke B, Pays E, Muyldermans S, and De Baetselier P. African trypanosomiasis therapy with nanobody conjugated human trypanolytic factor. Nature Med. 2006;12:580-4.

2. Cortez-Retamozo V, Backmann N, Senter PD, Wernery U, De Baetselier P, Muyldermans S, Revets $\mathrm{H}$. Efficient cancer therapy with a nanobody-based conjugate. Cancer Research. 2004;64:2853-7.

3. Hmila I, Saerens D, Ben-Abderrazek R, Vincke C, Abidi N, Benlasfar Z, Dabbek H, El Ayeb M, BouhaoualaZahar B, Muyldermans S. Effective neutralisation of whole scorpion venom by a bispecific Nanobody. Faseb J. 2010;24:3479-89.

4. Muyldermans S, Baral TN, Cortez-Retamozo V, De Baetselier P, De Genst E, Kinne J, et al. Camelid immunoglobulins and nanobody technology. Vet. Immunol. Immunopath. 2009;128:178-83.

5. Rothbauer U, Zolghadr K, Muyldermans S, Schepers A, Cardoso MC, Leonhardt H. A versatile nanotrap for biochemical and functional studies with fluorescent fusion proteins. Mol Cell Proteomics. 2009;7:282-9.

6. Rothbauer U, Zolghadr K, Nowak D, Tillib S, Schermelleh L, Gahl A, Backmann N, Conrath K, Muyldermans S, Cardoso MC, Leonhardt H. Targeting and tracing of antigens in living cells. Nat Methods. 2006;3:887-9.

7. Vaneycken I, Devoogdt N, Van Gassen N, Vincke C, Xavier C, Wernery U, Muyldermans S, Lahoutte $\mathrm{T}$, Caveliers V. Preclinical screening of anti-HER2 nanobodies for molecular imaging of breast cancer. Faseb J 2011;5:April 8, online .

\section{Recommended readings}

1. Kirchhofer A, Helma J, Schmidthals K, Frauer C, Cui $\mathrm{S}$, Karcher $\mathrm{A}$, et al. Modulation of protein properties in living cells using nanobodies. Nature Struct Mol Biol. 2010;17:133-8.

2. Saerens D, Huang L, Bonroy K, Muyldermans S. Antibody fragments as probe in biosensor and microarray development. Sensors. 2008;8:4669-86.

3. Saerens D, Hassanzadeh GH, Muyldermans S. Single domain antibodies as building blocks for novel therapeutics. Curr Opin Pharmacol. 2008;8:600-8.

\title{
La técnica MABA (Multiple Antigen Blot Assay): una aproximación e bajo costo al diagnóstico simultáneo de múltiples enfermedades infecciosas
}

\author{
Oscar Noya, Sandra Losada, Marilyan Toledo, Henry Bermúdez, María A. Lorenzo, \\ Adriana Gauna, Belkisyolé Alarcón de Noya
}

\begin{abstract}
Secciones de Biohelmintiasis e Inmunología, Instituto de Medicina Tropical, Facultad de Medicina, Universidad Central de Venezuela, Caracas, Venezuela
\end{abstract}

\section{Introducción}

La presencia simultánea de distintos agentes infecciosos en un mismo individuo es la condición habitual en los seres vivos en quienes estos organismos se puedencomportar como comensales o patógenos, dependiendo del estado inmunitario del huésped. Esta situación es frecuente en los países en desarrollo y es de especial relevancia para el personal de salud que requiere conocer la exposición actual o pasada a agentes infecciosos, que pudieran estar incidiendo en la patología motivo de su consulta médica.

Más allá de resolver el problema puntual de cada paciente, debe aprovecharse la oportunidad para obtener toda la información relativa a su condición infecciosa presente o pasada que de diagnosticarse tempranamente, pudiera evitar complicaciones e inclusive la muerte. Al no aprovechar esa consulta estaríamos ante la "oportunidad perdida", denominación frecuente entre los pediatras.
Para ello, se requiere disponer de técnicas de multidiagnóstico que permitan identificar simultáneamente la presencia de anticuerpos contra los agentes causales, sus antígenos o sus ácidos nucleicos.

Existen, al menos, tres tecnologías disponibles y entre ellas la técnica inmunocromatográfica MABA (Multiple Antigen Blot Assay), la cual permite evaluar simultáneamente el reconocimiento de hasta 28 antígenos diferentes, en una tira de nitrocelulosa $(1,2)$. La plasticidad de esta técnica estriba en permitir múltiples aplicaciones más allá del diagnóstico de agentes infecciosos, como es la evaluación de antigenicidad e inmunogenicidad de antígenos crudos, recombinantes, péptidos sintéticos, alérgenos y moléculas asociadas a fenómenos de autoinmunidad, entre otras.

En el presente caso, el énfasis está orientado al diagnóstico simultáneo de enfermedades infecciosas y parasitarias, tales como esquistosomiasis, 
enfermedadde Chagas, leishmaniasis, toxocariasis, toxoplasmosis, hepatitis viral, HIV, histoplasmosis y leptospirosis. Otros agentes microbianos están en proceso de evaluación.

La meta es que la técnica sea utilizada en los centros de salud como una prueba de tamización general, a fin de conocer el perfil serológico de los principales agentes infecciosos de cada consultante. Esta técnica es adaptable a formatos especiales (fiebres hemorrágicas, infecciones por descartar en bancos de sangre y trasplantes, mujeres embarazadas, enfermedades eruptivas de la infancia, etc.).

\section{Materiales y métodos}

Sueros de pacientes: se obtuvieron sueros de laboratorios de referencia de las diferentes enfermedades señaladas previamente, y de muestreos de poblaciones en áreas endémicas de parasitosis específicas. Se obtuvo el consentimiento informado de cada participante en este estudio. Cada caso se evaluó con las técnicas de referencia aceptadas internacionalmente, parasitológicas o inmunológicas (ELISA, inmunofluorescencia indirecta y Western blot).

Antígenos: se utilizaron antígenos crudos $(n=7)$, fracciones de los mismos $(n=3)$ y péptidos sintéticos $(n=4)$ obtenidos de proteínas relevantes de diferentes agentes infecciosos mediante la estrategia t-boc (3).

Metodología del MABA: se utilizó la metodología convencional de sensibilización del papel de nitrocelulosa con los diferentes antígenos con concentraciones variables entre 5 y $20 \mu \mathrm{g}$ por $\mathrm{ml}$ y su revelado con sustratos luminiscentes y cromogénicos $(1,2)$. El registro y el análisis cualitativo y cuantitativo se realizó con un analizador de imágenes (Chemidoc $\square$, BioRAD). Hasta ahora, el MABA ha sido utilizado como un método cualitativo, pero al utilizar el analizador de imágenes se convierte en una herramienta que permite el análisis cuantitativo del reconocimiento a las moléculas antigénicas en evaluación.

La sensibilidad y la especificidad de cada uno de los antígenos se estableció de forma individual al comparar los resultados de cada antígeno, con las pruebas de referencia.

\section{Resultados y conclusiones}

La sensibilidad y la especificidad obtenidas con los diferentes antígenos evaluados en esta serie fue variable ya que dependió de cada antígeno. De forma sucinta, los resultados obtenidos hasta ahora, muestran sensibilidades superiores al $75 \%$, teniendo sólo problemas con la especificidad correspondiente a los antígenos de Toxocara canis, Histoplasma capsulatum y Leptospira spp. que no se han podido minimizar eliminando los epítopos "glicosilados" con metaperiodato de sodio.

En cuanto al costo de evaluación de cada antígeno y tomando sólo en cuenta los materiales, es de US $\$ 0,07$ para MABA frente a US $\$ 0,114$ para la técnica de ELISA convencional. Si se añadiera el costo del personal, al realizarse en el caso del MABA en un tiempo comparable al de un solo agente causal por ELISA, el costo final dependería del número de antígenos incluidos en MABA, siendo significativamente menor que la prueba de ELISA, que es considerada la más económica de las técnicas de inmunodiagnóstico.

Con el fin de ajustar el método a un sistema automatizable, se ha iniciado la exposición de los sueros de forma cruzada utilizando el Miniblotter $^{\mathrm{TM}}$, bajo un formato que denominamos "Cross-MABA", el cual mantiene las mismas cualidades del MABA convencional, pero es más simple y rápida su ejecución, ya que no requiere el corte ni la manipulación de las tiras del papel de nitrocelulosa.

En conclusión, se reafirman las virtudes de una técnica aplicable en diferentes formatos y en diferentes instituciones de salud y las limitaciones observadas con algunos antígenos, como el de $T$. canis, que requiere la preadsorción de los sueros con antígeno de Ascaris suum, debido a la alta reactividad cruzada del mismo, que no permite su aplicación para estudios de poblaciones, pero sí para el diagnóstico individual de los casos de toxocariasis.

La alta especificidad y sensibilidad obtenida con losantígenos de Entamoebahistolytica, Toxoplasma gondii, Fasciola hepática, Trypanosoma cruzi, Schistosoma mansoni, cisticerco de Taenia solium y pé2ptidos de los virus HIV y VHC los reafirma para futuros estudios. Deben mejorarse algunos péptidos de HIV, S. mansoni y los antígenos crudos de Histoplasma capsulatum, Toxocara canis, Leptospira spp. y Leishmania mexicana.

La simplificación y el análisis cuantitativo mediante su registro por el analizador de imágenes, permiten un análisis más objetivo de los resultados y más factible de establecerse como un sistema automatizado, fácilmente adaptable a diferentes formatos y a los requerimientos de la demanda del sector salud.

Financiado por: Proyecto Multidiagnóstico (Proyecto No. UCV-G-2005000387), Misión Ciencia del FONACIT (Subproyecto 1-Proyecto No. 2007001425), Venezuela 


\section{Referencias}

1. Noya $O$, Alarcón de Noya B. The multiple antigen blot assay (MABA): a simple immunoenzymatic technique for simultaneous scrreening of multiple antigens. Immunol Letters. 1998;63:53-6.
2. Noya O, Losada S, Toledo M, Alarcón de Noya B. The multiple antigen blot assay-MABA, a simple, versatile and multi-purpose immunoenzymatic technique. En: Protein blotting. Methods in molecular biology. New York: The Humana Press Inc., The Humana Press: 2009. p. 237-51.

\title{
Low-stringency single-specific primer PCR as a molecular marker to evaluate genetic polymorphisms in Leishmania (Viannia) braziliensis rom cutaneous and mucosal leishmaniasis patients
}

\author{
Raquel S. Pacheco ${ }^{1}$, Fernanda S. Oliveira ${ }^{1}$, Cibele Baptista ${ }^{2}$, Armando Schubach ${ }^{2}$ \\ 1 Instituto Oswaldo Cruz, Rio de Janeiro, Brazil \\ 2 Instituto de Pesquisas Clínicas Evandro Chagas, Rio de Janeiro, Brazil
}

\section{Introduction}

Leishmania (Viannia) braziliensis is the main widespread species responsible for human cases of American tegumentary leishmaniasis in Brazil. The disease is characterized by chronicity, latency, and metastatic tendencies. In some cases, after the appearance of an initial cutaneous lesion, mucosal involvement can occur as a consequence of the dissemination of the parasite through the blood and lymphatic systems.

\section{Materials and methods}

We have used polymerase chain reaction (PCR) and low-stringency single-specific primer PCR (LSSP-PCR) analyses to detect $L$. (V.) braziliensis and investigate genetic polymorphisms in kDNA minicircles of parasite populations present in cutaneous, oral and nasal mucosa lesions from 54 patients with American tegumentary leishmaniasis, including HIV co-infected subjects, proceeding from Rio de Janeiro, Brazil.

\section{Results and discussion}

The intraspecific polymorphism of the variable region of $L$. (V.) braziliensis kDNA minicircles has been investigated by our group using LSSP-PCR. Similar genotypes were observed in parasites recovered from nasal and oral mucosa lesions of the same patient. In contrast, genetically divergent profiles were found in recurrent lesions from patients biopsied at different times within a period of 1 year, suggesting the polyclonality of the initial inoculum (1).

We have also compared panels of samples obtained from cutaneous lesions of patients with typical and atypical clinical manifestations of the disease. Nine different genotypes were identified and phenetic analysis has grouped the isolates into two major clusters. A prevalent genotype was detected in the largest number of samples investigated.
Although no association had been observed between genotypes and clinical manifestations of the disease, two different genotypes could be identified in the initial and reactivated lesions of the same patient (2).

In order to evaluate the impact of immunosuppression on the genetic structure of the parasite, the genetic polymorphism of $L(V$.) braziliensis detected in cases of mucosal leishmaniasis from HIV-infected and non-HIV-infected patients has been evaluated. Samples obtained from human cases of leishmania/HIV co-infection were found to be genetically divergent. Phenetic analysis grouped the genetic profiles into two distinct clusters, which discriminated between samples obtained from HIVinfected and non-HIV-infected patients (3).

The clinical and epidemiological implications of such results will be discussed.

\section{References}

1. Oliveira FS, Valete-Rosalino CM, Schubach A, Pacheco RS. KDNA minicircle signatures of Leishmania (Viannia) braziliensis in oral and nasal mucosa from mucosal leishmaniasis patients. Diagn Microbiol Infect Dis. 2010;66:361-5.

2. Baptista C, Schubach A, Madeira MF, Leal CA, Pires $\mathrm{MQ}$, Oliveira FS, Conceição-Silva F, Rosalino CM, Salgueiro MM, Pacheco RS. Leishmania (Viannia) braziliensis genotypes identified in lesions of patients with atypical or typical manifestations of tegumentary leishmaniasis: evaluation by two molecular markers. Exp Parasitol. 2009;121:317-22.

3. Oliveira FS, Valete-Rosalino CM, Schubach A, Madeira MF, Pacheco RS. Genetic polymorphism in Leishmania (Viannia) braziliensis detected in mucosal leishmaniasis of HIV-infected and non-HIVinfected patients. Trans Roy Soc Trop Med Hyg. 2011; in press.

Support: CNPq, FAPERJ, and Instituto Kinder do Brasil 